\title{
Iron Deficiency Alters Auditory Recognition Memory in Newborn Infants of Diabetic Mothers
}

\author{
ASHAJYOTHI M. SIDDAPPA, MICHAEL K. GEORGIEFF, SANDI WEWERKA, CATHY WORWA, \\ CHARLES A. NELSON, AND RAYE-ANN DEREGNIER
}

\begin{abstract}
Division of Neonatology, Department of Pediatrics [A.M.S., M.K.G., C.A.N.], Institute of Child
Development [M.K.G., S.W., C.A.N.], and Center for Neurobehavioral Development [M.K.G., S.W., C.A.N.], University of Minnesota, Minneapolis, MN 55455, U.S.A.; Division of Neonatology [C.W.], Children's Hospital-St. Paul, St. Paul, MN 55102, U.S.A.; and Division of Neonatology, Department of Pediatrics [R.-A.d.R.], Northwestern University Feinberg School of Medicine \& Children's Memorial Hospital, Chicago, IL 60611, U.S.A.
\end{abstract}

\begin{abstract}
ABSTR
Infants of diabetic mothers (IDMs) are at risk for perinatal
brain iron deficiency that may target the developing hippocam-
pus. The objective of this study was to evaluate hippocampally
based recognition memory and infant development in IDMs with
suspected brain iron deficiency (BID; cord ferritin $\leq 34 \mu \mathrm{g} / \mathrm{L}$ )
compared with IDMs with sufficient brain iron stores (BIS; cord
ferritin $>34 \mu \mathrm{g} / \mathrm{L}$ ) using event-related potentials (ERPs). ERPs
assessed neonatal auditory cortical responses to sounds and
auditory recognition memory in response to the mother's voice
compared with a stranger's voice. Thirty-two newborn IDMs had
cord serum ferritin concentrations and provided neonatal ERP
data ( $n=23$ ) and/or blinded 1 y developmental assessments (
$=28$ ). Auditory cortical responses to speech and nonspeech
sounds were similar in the BID and BIS groups. In the maternal
voice recognition paradigm, peak latencies were shorter in the
BID group than in the BIS group. Infants in the BIS group
displayed a significant negative slow wave for the strangers'
\end{abstract}
Iron plays a significant role in early brain structural development and function because it forms an important component of enzymes involved in cell replication, myelinogenesis, neurotransmitter synthesis, and cellular energy metabolism $(1,2)$. Deficiency of dietary iron during infancy and childhood is associated with long-term effects on cognitive development that persist after treatment of iron deficiency (3). Iron deficiency also may occur earlier in life, during the fetal period, in infants of diabetic mothers (IDMs) and infants with intrauterine growth restriction (4-7). When severe, fetal iron deficiency has been associated with a $40 \%$ reduction in brain iron con-

Received October 7, 2003; accepted February 18, 2004.

Correspondence: Raye-Ann deRegnier, M.D., Room 404B, Prentice Women's Hospital, 333 E. Superior St., Chicago, IL 60611, U.S.A.; e-mail: r-deregnier@northwestern.edu

Supported by National Institutes of Health Grants NS-32755 (to M.K.G., C.A.N., and R.-A.d.Rf) and HD-29421 (to M.K.G. and C.A.N.).

DOI: 10.1203/01.pdr.0000127021.38207.62 voices compared with the mothers' voices, whereas the BID group did not. Higher cord ferritin concentrations were correlated with larger negative slow waves at the right temporal (T4) electrode site. At 1 y of age, motor development was slower in the BID group than in the BIS group. IDMs suspected to have BID demonstrated impaired neonatal auditory recognition memory and lower psychomotor developmental scores at $1 \mathrm{y}$ of age than IDMs with BIS. These impairments map onto areas of the developing brain known to be vulnerable to iron deficiency. (Pediatr Res 55: 1034-1041, 2004)
BID, brain iron deficient
BIS, brain iron sufficient
ERP, event-related potential
IDM, infant of a diabetic mother

Abbreviations centration in the human IDM (6). In perinatal animal models, a similar degree of iron deficiency results in diminished hippocampal iron content and decreased cytochrome $c$ oxidase activity (8), with persistent hippocampal biochemical (9) and dendritic structural changes (10).

Perinatal reductions in iron content in the hippocampus may have functional consequences for the development of recognition memory, beginning soon after birth. In the newborn, the degree of histologic maturation of the hippocampus seems to be sufficient to support rudimentary recognition memory abilities (11). Indeed, neonatal recognition memory has been demonstrated using behavioral and neurophysiologic techniques (12-15). Therefore, fetal insults that affect the developing hippocampus may cause in recognition memory measurable impairments that can be assessed during the neonatal period. We have previously shown that IDMs have impairments in auditory, visual, and cross-modal recognition memory at birth and during the first 8 mo of postnatal life $(14,16,17)$. 
However, the role of iron deficiency in these findings has not been explored.

The purpose of this study was to evaluate auditory recognition memory in newborn IDMs with evidence of fetal iron deficiency severe enough to cause brain iron deficiency (BID) and to compare infant development at $1 \mathrm{y}$ of age in the BID and brain iron sufficient (BIS) groups. We hypothesized that newborn IDMs with suspected BID would show evidence of impaired maternal voice recognition at birth and lower developmental scores at $1 \mathrm{y}$ of age compared with IDMs with normal iron status. Maternal voice recognition was evaluated using event-related potentials (ERPs), a neurophysiologic technique that is widely used by cognitive neuroscientists to study recognition memory.

\section{METHODS}

Subjects. The study was approved by the Institutional Review Boards of the three participating hospitals. Informed consent was obtained from each family. Fetal metabolic effects of diabetes are seen in infants of both gestational and pregestational diabetic mothers (5). Therefore, both groups were recruited to participate in a longitudinal study of the effects of maternal diabetes on infant memory development. As it was anticipated that some of the diabetic women would deliver before term, premature infants who were born at or beyond 34 wk gestation were included in the study when they were healthy after birth without complications of prematurity.

The iron status of the newborn human brain cannot be measured noninvasively but can be inferred from the serum ferritin concentration at birth. Brain iron concentrations correlate directly with liver iron concentrations ( $r=0.8$ in IDMs) (6), and brain iron is preserved until liver storage iron is decreased by $70 \%(6,18)$. Neonatal liver iron stores per kilogram of body weight can be estimated from the newborn serum ferritin concentration on the basis of the logarithmic relationship between the two factors (19). Seventy percent loss of normal neonatal storage iron results in a value of $5.1 \mathrm{mg}$ $\mathrm{Fe} / \mathrm{kg}$ body weight and predicts a serum ferritin concentration of $34 \mu \mathrm{g} / \mathrm{L}$ (19). Therefore, a neonatal serum ferritin concentration of $34 \mu \mathrm{g} / \mathrm{L}$ was used as the threshold value for determining suspected brain iron deficiency in this study.

Umbilical cord or neonatal blood (obtained in the first $3 \mathrm{~d}$ of life) was obtained for evaluation of serum ferritin concentrations, determined in duplicate by chemoluminescent immunoassay (Beckman Access Immunoassay System; Beckman Coulter, Brea, CA, U.S.A.) $(4,5)$. Interassay variability was $<3 \%$. Infants were divided into two groups on the basis of their ferritin concentrations. Ferritin concentrations of $\leq 34$ $\mu \mathrm{g} / \mathrm{L}$ classified an infant as suspected BID, with ferritin concentrations of $>34 \mu \mathrm{g} / \mathrm{L}$ as BIS.

For comparing the groups for perinatal complications not related to diabetes, an obstetrics complication scale was used (20). Points given for diabetes or insulin use were removed before comparison of the groups. Hearing was screened by a pediatric audiologist using an otoacoustic emissions test. Infants who failed the screening evaluation were excluded from the study. Neonatal ERPs were collected as in previous studies $(14,15)$, summarized below.

ERP background information. ERPs are neurophysiologic recordings used by cognitive neuroscientists to evaluate abilities such as memory. In an ERP, the recording of the EEG is time-locked to multiple presentations of the stimuli. Stimuli that have similar physical properties but differ in cognitive aspects such as familiarity are chosen. By averaging data from multiple stimulus presentations together, random brain activity that is not related to the stimulus is decreased and brain activity that occurs in response to the stimulus emerges in the waveform (21). Individual infants' ERPs can be averaged together to create a grand average ERP.

The infant ERP waveform consists of peaked components and slow waves. Peaked components, or peaks, are deflections in the brain activity that represent the synchronized activation of discrete populations of neurons. Peaks rise from the baseline and fall back over a short period. The amplitude is measured at the point of maximal deflection. The latency is measured as the time from the onset of the stimulus to the maximal deflection (Fig. 1).

The peaks generated in the auditory cortex undergo a distinct maturational pattern (22). The most immature response (level I) is typically seen before term gestation. It consists of negative peaks over midline and lateral scalp locations process (Fig. 1). With maturation, the peaked component becomes positive first over the midline and finally over the lateral scalp site (level V). By term, most infants have maturity levels of level III or greater. The positive peak noted over the midline is denoted as the $\mathrm{P} 2$ peak as it is thought to be a predecessor of the adult P2 peak (23).

In contrast to the peaks, slow waves are slow deflections in the ERP without discernible peaks (Fig. 2). Slow waves reflect diffuse and sustained neural activity (21) and are commonly seen in very young infants. Slow waves are analyzed by integrating the area under the ERP curve. We have previously demonstrated negative slow waves in response to a stranger's voice in comparison with the mother's voice in healthy fullterm infants $(14,15)$ but not in IDMs $(14)$. However, the role of iron deficiency was not assessed in that study.

ERP study design. Infants were tested at $38-42$ wk postmenstrual age, while in a behavioral state of active sleep (24). Two paradigms were administered. First, auditory cortical maturation was assessed using ERP methods similar to those described by Novak et al. (22). Stimuli were a speech sound (the sound "ba" in a female voice) and a nonspeech sound (a computer chime). Both sounds were recorded, digitized, and edited to $400 \mathrm{~ms}$ and $80 \mathrm{~dB}$ sound pressure level using the Sound Blaster software program (Creative Labs; Milpitas, CA, U.S.A.). The sounds were presented alternately for 100 trials while brain activity was recorded as described below.

Second, to assess auditory recognition memory processing, ERPs were recorded in response to the mother's voice alternating with a stranger's voice for 100 trials (i.e. mother-stranger-motherstranger). The stranger's voice varied for each infant and was the mother's voice from the previous infant. The stimulus consisted of the word "baby" digitized and edited to $750 \mathrm{~ms}$ and $80 \mathrm{~dB}$ sound pressure level, again using the Sound Blaster software program. It is not likely that newborn infants can recognize specific words, 
Maturity

Level I

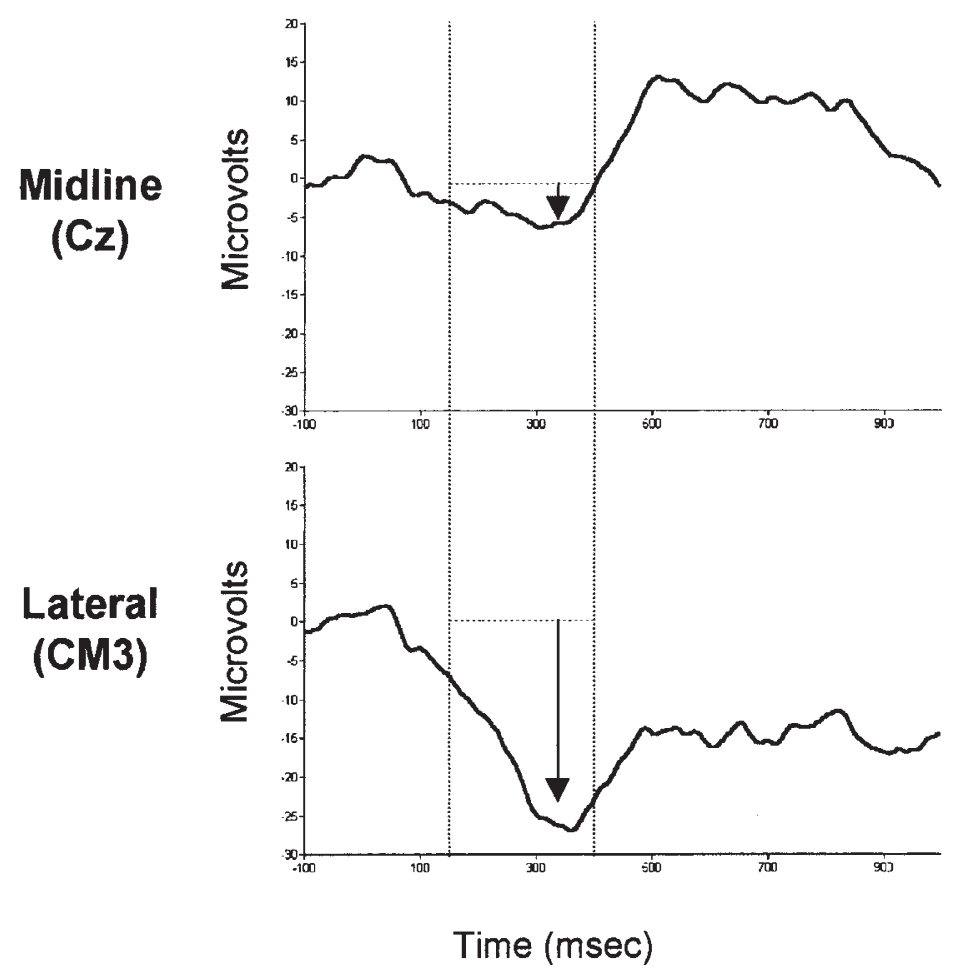

Maturity

Level V

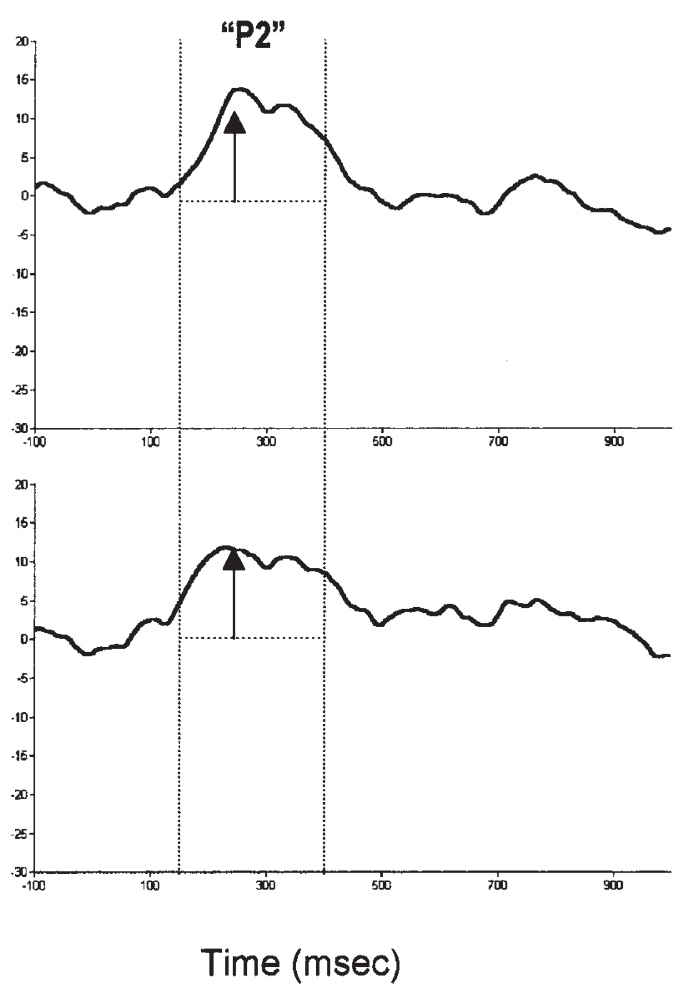

Figure 1. Examples of individual ERPs from infants at maturity level I and maturity level V, demonstrating development of the auditory cortical response. Vertical dashed lines indicate the window of analysis for the peaks $(150-400 \mathrm{~ms})$. Horizontal dashed lines indicate the baseline. Arrows show deflections of peak from baseline: negative for the midline and lateral sites in level I and positive at both sites for level V. The midline positive peak is denoted P2 as it is thought to be the predecessor of the adult P2 peak.

but it was presumed that all pregnant women and new mothers would have used the word "baby."

All sounds were presented to the infant's right ear using an EAR tone 3A-insert earphone (Cabot Corporation; Indianapolis, IN, U.S.A.). The right ear was chosen because there is a right ear advantage in processing language (25). Stimulus presentation was computerized, and the technician who performed the ERP test was not able to hear the sounds during the test session.

ERP recordings. For evaluating auditory cortical maturation, ERPs were recorded from the midline central $(\mathrm{Cz})$ site and from two lateral sites denoted CM3 and CM4 $(14,15,22)$. For evaluating recognition memory, ERPs were recorded from parietal, central, and frontal midline sites $(\mathrm{Pz}, \mathrm{Cz}$, and $\mathrm{Fz})$ as well left and right anterior temporal sites (T3 and T4), according to the International 10/20 system of electrode placement (26). Data were recorded for $100 \mathrm{~ms}$ prestimulus (baseline) and for $2000 \mathrm{~ms}$ after the stimulus onset. The interval between the stimuli varied randomly from 3900 to $4900 \mathrm{~ms}$. The raw EEG was recorded, artifacts were rejected, and average ERPs were created via computer algorithms using methods described by Nelson et al. (21).

At $1 \mathrm{y}$ of age (adjusted for prematurity if needed), the Mental and Psychomotor Indices of the Bayley Scales of Infant Development (II) (27) were administered by an occupational therapist who was blinded to the group assignment of the infant.

Data analysis. Each infant's speech and nonspeech sound ERPs were assigned maturity levels I-V (22). P2 peak amplitudes and latencies were measured for the peak in the window of 150-400 $\mathrm{ms}$ at the $\mathrm{Cz}$ and Fz electrode sites (Fig. 1). For the ERPs elicited by the mothers' and strangers' voices, the P2 peaks and latencies also were evaluated as above. The slow wave activity was analyzed by integrating the areas under the curve (21). This was done for the mother and stranger ERPs at each of the five scalp electrode sites ( $\mathrm{Pz}, \mathrm{Cz}, \mathrm{Fz}, \mathrm{T} 3$, and T4), over the entire 2000-ms recording interval.

Statistical analysis. Statistics were performed using the Statview 4.5/SuperAnova software package (Abacus Concepts, Berkeley, CA, U.S.A.). Clinical characteristics were compared between the groups using two-tailed $t$ tests or Fisher's exact tests. Maturity scores for the speech and nonspeech sound stimuli were compared using Fisher's exact tests. The P2 peak amplitudes and latencies and areas under the curve (for slow wave activity) were analyzed using repeated measures ANOVA with brain iron group as a factor. Significant differences were followed up with Newman-Keuls multiple range tests or paired $t$ tests as appropriate.

For each infant, the area under the curve for the mother's voice ERP was subtracted from the area under the curve for the 


\section{BIS Group}

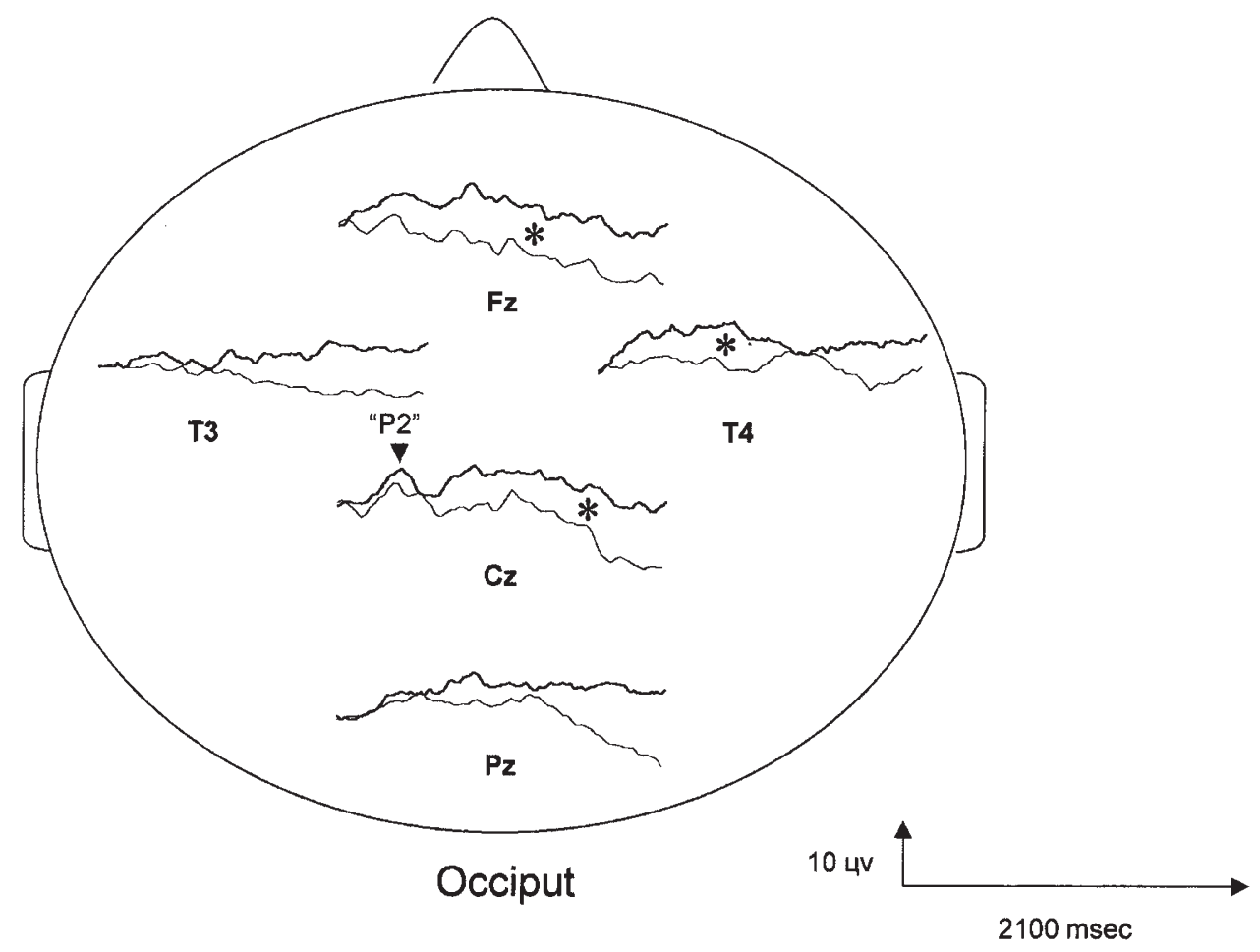

Figure 2. Grand average ERPs for the BIS group in response to the mother's voice (thick line) and stranger's voice (thin line) over the five electrode sites, drawn on a cartoon of the head. Note the slow negativity occurring in response to the stranger's voice, indicated by * at electrode sites where the mother's voice ERP is significantly different from the stranger's voice ERP. The P2 peak is marked at the Cz electrode site.

stranger's voice ERP to assess the area between the two curves, or the area of the slow wave itself. Neonatal ferritin concentrations were correlated with the areas of the slow waves at each of the five electrode sites (Pz, Cz, Fz, T3, and T4) using linear regression analyses.

Data are presented as the mean \pm one SEM. A $p<0.05$ was considered to be statistically significant.

\section{RESULTS}

Thirty-four infants had umbilical cord or neonatal serum ferritin concentrations, met the inclusion criteria, and participated in the study. For two infants, the neonatal ERPs were not analyzable because of excessive artifacts in the recordings. These two infants withdrew from the study before $1 \mathrm{y}$ of age, so their data were not used in any analyses. Of the remaining 32 infants, 10 had suspected BID with serum ferritin concentrations $\leq 34 \mu \mathrm{g} / \mathrm{L}$ and the remaining 22 infants composed the BIS group. Twenty-three newborns provided artifact-free ERPs (9 BID, 14 BIS), and 28 infants provided 1-y follow-up (9 BID, 19 BIS). Data from 11 (3 $\mathrm{BID}, 8 \mathrm{BIS}$ ) of the infants was previously published as part of a larger study in which ferritin concentrations were not available for the majority of the infants (14).

Clinical characteristics of the BID and BIS groups are shown in Table 1. Mean birth weights of the BID group were significantly higher than those of the BIS infants. Pregestational diabetes was more common in the BID group, but insulin therapy was common in both groups. Obstetric complications apart from diabetes were similar between the groups. The BIS and BID groups underwent ERP testing at the same postnatal and postmenstrual ages.

Auditory cortical responses to speech and nonspeech sounds. For the speech and nonspeech sounds, 8 BID and 13 BIS infants provided artifact-free data. One BID infant had an atypical ERP with no discernible peaks in response to either the speech or the nonspeech sound, and maturity levels could not be assigned. For the remaining infants, there were no differences between the groups in the proportions of infants at level $\mathrm{V}$ maturity for the speech sound

Table 1. Perinatal characteristics of the BID and BIS groups

\begin{tabular}{lcc}
\hline & BID $(n=10)$ & BIS $(n=22)$ \\
\hline Gestational age $(\mathrm{wk})$ & $38.5 \pm 0.3$ & $38.4 \pm 0.3$ \\
Premature $[<37 \mathrm{wk} ; n(\%)]$ & 0 & $2(9 \%)$ \\
Birthweight $(\mathrm{g})$ & $4357 \pm 233$ & $3466 \pm 132 *$ \\
Birthweight $>90$ percentile $[n(\%)]$ & $8(80)$ & $8(36) \dagger$ \\
Range of ferritin concentrations & $5-30$ & $42-236$ \\
$\quad(\mu \mathrm{g} / \mathrm{L})$ & & \\
Obstetric risk score & $4.9 \pm 0.8$ & $5.3 \pm 0.4$ \\
Pregestational diabetes $[n(\%)]$ & $7(70)$ & $(27)^{*}$ \\
Treated with insulin [n $(\%)]$ & $8(80)$ & $13(59)$ \\
Postmenstrual age at ERP test $(w k)$ & $40.8 \pm 0.4$ & $40.9 \pm 0.3$ \\
Age at ERP test $(\mathrm{d})$ & $15.3 \pm 3.5$ & $15.8 \pm 3.5$ \\
\hline
\end{tabular}

Data are presented as mean + SEM or number (\%).

$* p<0.05$.

$\dagger p$ value $=0.054$ 
(BID 6 of 7; BIS 11 of 13) or for the nonspeech sound (BID 5 of 7 ; BIS 13 of 13; Fig. 3).

There were no differences between the BID and BIS groups in the P2 peak amplitudes and latencies for the speech and nonspeech sounds in the window $150-400 \mathrm{~ms}$. Peak amplitudes were higher at the central $(\mathrm{Cz})$ electrode site $(10.9 \pm 1$ $\mu \mathrm{V})$ than at the frontal $(\mathrm{Fz})$ electrode site $(9.2 \pm 1.1 \mu \mathrm{V} ; p=$ 0.05 ), a typical pattern for this age group, and this was seen across both the BID and the BIS groups.

Recognition memory. Nine infants in the BID group and 13 in the BIS group provided artifact-free data for the recognition memory paradigm. The $\mathrm{P} 2$ peak amplitude for the ERP elicited by the mother's voice was similar to that elicited by the stranger's voice for both of the groups. There were no group differences for peak amplitudes. There were no significant interactions (Figs. 2 and 4).

Across both the mother's and the stranger's voice ERPs, latencies to the P2 peak were significantly shorter in the BID group than in the BIS group (BID $247 \pm 12.7 \mathrm{~ms}$; BIS $290 \pm$ $10.5 \mathrm{~ms} ; p=0.049)$. For both the BID and the BIS groups, the latency was longer to the peak elicited by the mother's voice (mother ERP latency $290 \pm 11.2 \mathrm{~ms}$; stranger ERP latency 255 $\pm 11.9 \mathrm{~ms} ; p=0.049$ ). There were no effects of electrode site or any significant interactions in the ANOVA.

The ANOVA for the areas under the curve showed a significant group $\times$ area interaction $(p=0.019)$. Post hoc testing revealed a significant difference between ERP areas under the curve for the mother's and stranger's voices $(p=0.014)$ in the BIS group but not in the BID group $(p=0.43)$. For the BIS group, the difference between the ERPs elicited by the mothers' and strangers' voices was a negative slow wave, a typical finding seen in healthy full-term newborns. This was a main effect, seen across the five electrode sites. Using paired $t$ tests, the mothers' and strangers' voices were significantly different at the central $(\mathrm{Cz})$, frontal $(\mathrm{Fz})$, and right temporal $(\mathrm{T} 4)$ electrodes sites (Table 2).

Linear regression analyses were conducted using the difference wave areas under the curve (area of the slow wave: stranger ERP area - mother ERP area) at each of the five electrode sites $(\mathrm{Pz}, \mathrm{Cz}, \mathrm{Fz}, \mathrm{T} 3$, and $\mathrm{T} 4)$ as the dependent variables and the neonatal ferritin concentration as the independent variable. The neonatal ferritin value was significantly correlated with the difference wave areas under the curve at the T4 electrode site $\left(R^{2}=0.296, p=0.009\right.$; Fig. 5$)$. The ferritin concentration was not correlated with difference wave areas under the curve at the other four electrode sites.

Twenty-eight infants had 1-y testing using the Bayley Scales of Infant Development. The mean Mental Developmental Index score was similar between the groups $(n=28$; BID $97 \pm$ 3 ; BIS $101 \pm 2$ ). Psychomotor Developmental Index scores were lower in the BID group than in the BIS group $(n=27$; BID $86 \pm 8$; BIS $103 \pm 3 ; p=0.03$ ).

\section{DISCUSSION}

Our study demonstrates that neonatal auditory recognition memory processing is impaired in iron-deficient newborns of diabetic mothers tested at term postmenstrual age, suggesting that the iron-deficient fetal environment altered the development and/or the function of the neural circuits underlying this process. Impairments were also detected in 1-y motor development at a time when it was likely that all if the infants were iron sufficient (28).

As with other nutrients that affect brain development, the brain processes that will be most affected by iron deficiency will depend on the timing, degree, and duration of the deficiency (2). For example, Roncagliolo et al. (29) hypothesized that delayed latencies on auditory brainstem evoked response in postnatally iron-deficient children are due to effects on myelination because the time of deficiency corresponded with a period of rapid myelin production. Similarly, Angulo-Kinzler et al. $(30,31)$ demonstrated abnormal motor activity in $6-$ to 12-mo-old postnatally iron-deficient infants and postulated that these effects may be mediated through developing dopaminergic striatal pathways. Given that many neural systems are in a different stage of development during late gestation (32), the effects of gestational/early postnatal iron deficiency are likely to differ from those seen with postnatal iron deficiency.

This study showed three major differences between the BID and BIS groups; an impaired ability to discriminate a familiar from a novel stimulus in the BID group, shorter ERP latencies in the BID group, and poorer motor function ability at $12 \mathrm{mo}$ of age in the BID group. These effects are consistent with the abnormalities in brain development noted in animal models of perinatal iron deficiency (33-35).

The ability to discriminate novel from familiar is a recognition memory behavior mediated by the intact hippocampus. The newborn infant demonstrates histologic, behavioral, and neurophysiologic evidence of a functional hippocampus at birth (11-15). In our study, the BID infants showed a marked

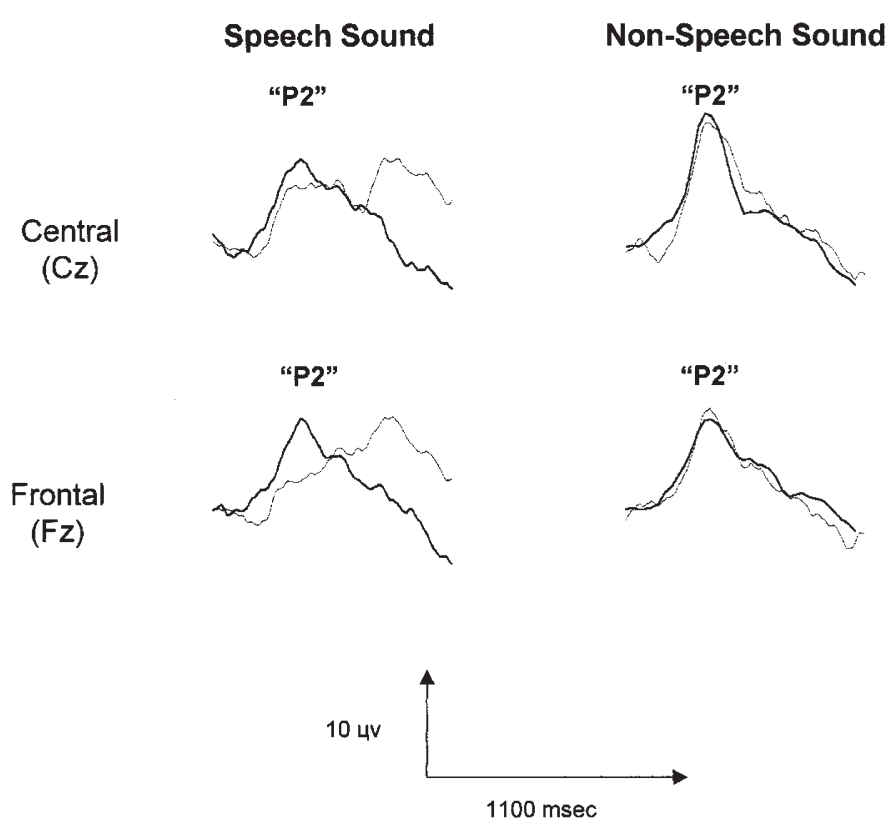

Figure 3. Grand average ERPs for the BID group (thin line) and BIS group (thick line) in response to the speech and nonspeech sounds, recorded at the midline central $(\mathrm{Cz})$ and frontal $(\mathrm{Fz})$ electrode sites. No significant differences were noted between the BID and BIS groups for peak amplitudes and latencies measured for the P2 peak at $150-400 \mathrm{~ms}(p=0.7)$. 


\section{BID Group}

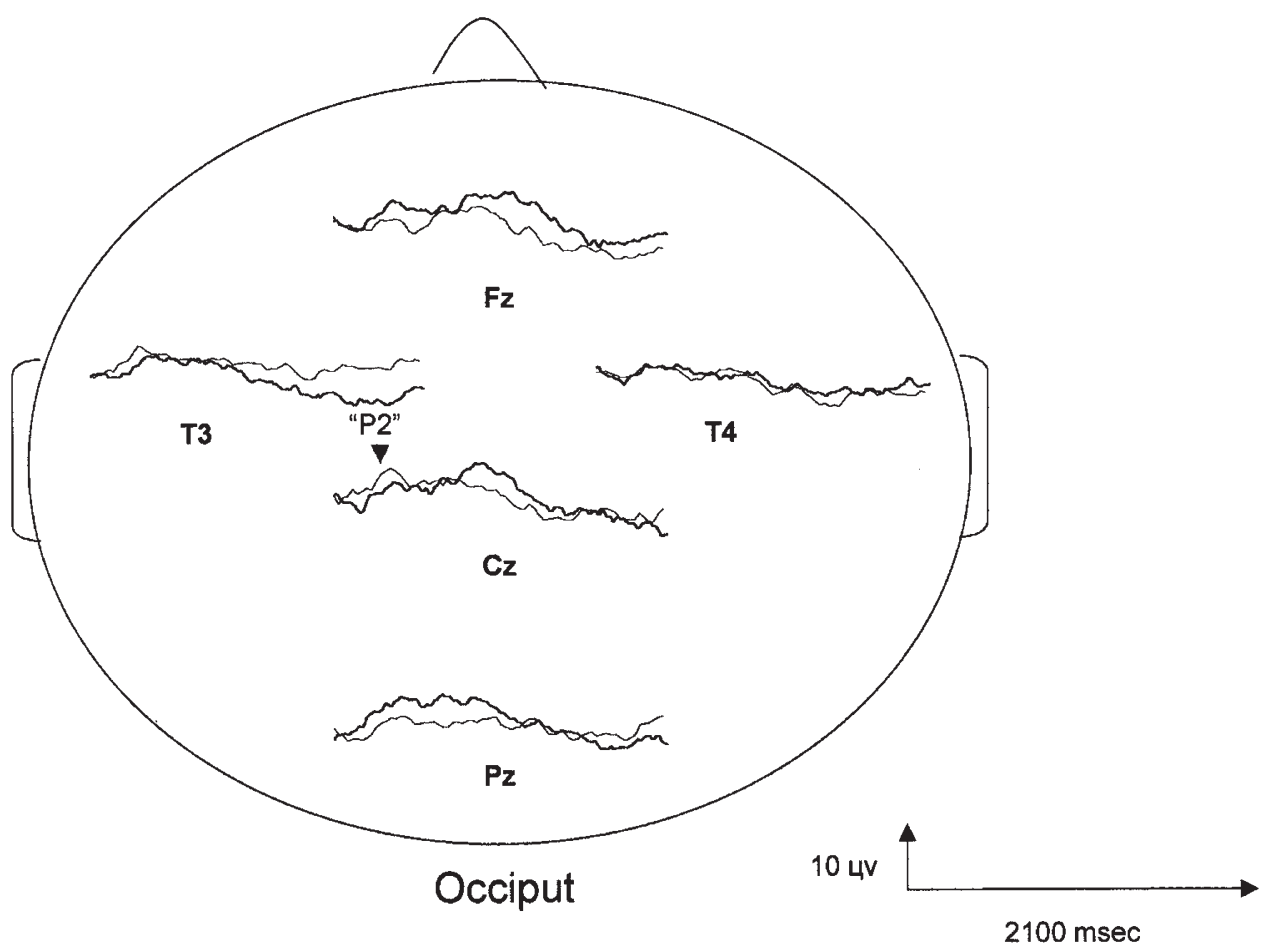

Figure 4. Grand average ERPs for the BID group in response to the mother's voice (thick line) and the stranger's voice (thin line) over the five electrode sites, drawn on a cartoon of the head. Note the lack of activity over the T4 electrode site.

attenuation in the negative slow wave that is prominent throughout the recording in the BIS group (Figs. 2 and 4). This was particularly prominent at the right temporal electrode site (T4), where the area of the negative slow wave correlated with the neonatal ferritin value (Fig. 5). This neurophysiologic evidence of an inability of the BID infants to discriminate the novel from the familiar stimulus could be due to structural or functional abnormalities in the hippocampally mediated brain pathways for recognition memory. Although no studies in iron-deficient human newborns have assessed the integrity of the hippocampus, rat models demonstrate several significant effects of iron deficiency. For example, a $40 \%$ decrease in brain iron impairs neuronal energy metabolism as indexed by cytochrome $c$ oxidase activity in hippocampal subarea CA1 (8) and by phosphocreatine:creatine ratio on magnetic resonance spectroscopy (9). Energy-dependent processes such as early dendritic elongation and subsequent proximal branch formation are delayed in the same model (10). Furthermore, hippocampal glutamate and GABA concentrations are chronically elevated in perinatal iron deficiency (9) with alterations in NMDA receptor subunit expression (10). These structural and biochemical changes may result in disruptions in synaptic efficacy, affecting the function of the developing hippocampus.

Differences in the latencies to the peak components of an ERP reflect transmission time in the nervous system and may be related to the density and efficacy of the synapses, the degree of myelination, and the complexity of the involved neural circuitry $(36,37)$. Prolonged latencies seen in older infants' auditory brainstem responses have been postulated to be due to impaired myelination of the brainstem (38). Impaired myelination has been described in rat models of early iron deficiency $(39,40)$. The shorter latencies noted in this study of BID neonatal IDMs are not likely to be explained by hypomyelination. First, the latencies to the peak were shorter in the BID group and not prolonged, as would be anticipated with hypomyelination. The P2 peak studied here is thought to

Table 2. Areas under the curve $\left(\mu \mathrm{V} / \mathrm{ms}^{2}\right)$ for the ERPs from the mother's and stranger's voices for the BID and BIS groups

\begin{tabular}{crrrr}
\hline & \multicolumn{2}{c}{ BID } & \multicolumn{2}{c}{ BIS } \\
\cline { 2 - 4 } Electrode site & Mother's voice & Stranger's voice & Mother's voice & Stranger's voice \\
\hline Pz & $716 \pm 3765$ & $5681 \pm 3692$ & $10692 \pm 4707$ & $-1499 \pm 4544$ \\
Cz & $-4015 \pm 3357$ & $2453 \pm 2172$ & $7862 \pm 4025$ & $-6309 \pm 3231^{*}$ \\
Fz & $413 \pm 3918$ & $-390 \pm 2440$ & $4063 \pm 4217$ & $-10104 \pm 5034^{*}$ \\
T3 & $-620 \pm 4157$ & $-328 \pm 2766$ & $2606 \pm 2164$ & $-2049 \pm 1932$ \\
T4 & $-3247 \pm 2948$ & $122 \pm 2546$ & $8549 \pm 2595$ & $-1863 \pm 2403^{*}$ \\
\hline
\end{tabular}

Data presented as the mean + SEM.

* Significant difference between areas under the curve for the ERPs elicited by the mothers' and strangers' voices using paired $t$ tests $(p<0.05$ ). 


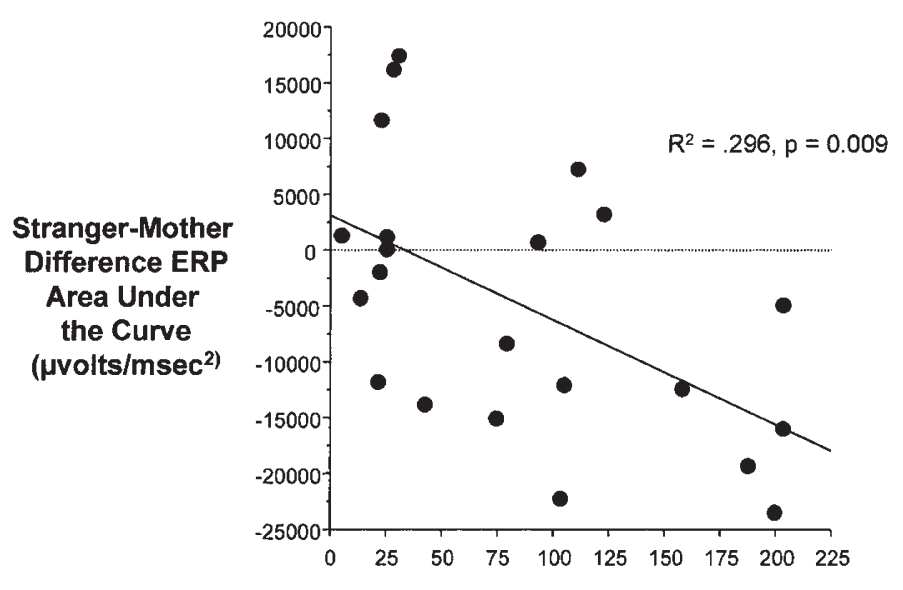

Ferritin $(\mu \mathrm{g} / \mathrm{L})$

Figure 5. Regression plot showing correlation between ferritin value ( $x$ axis) and difference in wave areas under the curve at the right temporal (T4) electrode site on the $y$ axis.

represent activation of auditory cortex, and at this stage of neuronal development, myelination is seen mainly in the brainstem and mid-brain in humans and thus is unlikely to affect hippocampal or auditory cortical function at birth $(32,36)$. Thus, differences in latencies are unlikely to be due to hypomyelination in the BID group. The faster latencies in the BID group are associated with decreased activity later in the recording (Figs. 2 and 4), so they may indicate a lack of sustained neural activity in the recognition memory circuitry, suggesting differences in the formation and function of axons, dendrites, and synapses as opposed to alterations in myelin production. Furthermore, significant differences in the ERPs were found only for the recognition memory task, and latency differences were not noted in the peaks elicited by the speech and nonspeech sounds. Although more subtle differences in sensory processing might be elicited with larger groups of infants, on the basis of the data presented here, we speculate that the primary problem may be within the recognition memory circuitry of the brain, including the hippocampus and other areas of the medial temporal lobe.

At $1 \mathrm{y}$ of age, the BID group showed lower Psychomotor Developmental Index scores than the BIS group. Motor function is dependent in part on the striatum, which is heavily innervated with dopaminergic fibers. Multiple studies in rats with early iron deficiency have shown impaired dopaminergic function mainly in the basal ganglia with decreased dopamine receptors, decreased uptake, and increased extracellular concentrations of dopamine (41-43). The striatum is important for regulating movement, and iron-deficient rats with altered dopamine status show significant movement disorders (44). Infants with postnatal iron deficiency have reduced motor activity that does not reverse with iron treatment $(30,31)$. Although the Bayley Scales of Infant Development do not directly assess striatal-based movements, impairments in that circuit could affect the performance on motor tasks in the inventory and account for the findings in our study group.

We speculate that prolonged difficulties with motor development seen in the BID group of this study may be conse- quences of perinatal iron deficiency. We did not obtain 1-y evaluations of iron status in this group of infants. However, a previous study of fetal iron deficiency indicated that the iron deficiency had resolved by $6-12$ mo, even among breast-fed infants who were not supplemented with iron (28). Thus, we speculate that the effect of perinatal iron deficiency continues beyond the period of deficiency. The finding of prolonged developmental consequences of fetal iron deficiency is consistent with data reported by Tamura et al. (45). They showed that infants who were born with ferritin concentrations in the lowest quartile performed more poorly on higher cognitive tests at school age than infants with ferritin concentrations $>25$ th percentile. Although postnatal iron deficiency occurs at a later phase of brain development, it is also associated with shortand long-term negative effects on the developing brain $(3,29$, $46,47)$.

In previous studies, children of diabetic mothers have been shown to have lower scores on cognitive testing, attention deficits, and impairment of gross and fine motor achievement compared with control children (48-50). These problems have been correlated with poor maternal glycemic control during pregnancy (49). The degree of iron deficiency in the newborn IDM is also a function of poor maternal glycemic control and to the degree of fetal hypoxia and augmented erythropoiesis (5). Studies in fetal sheep demonstrate that fetal hyperglycemia with its attendant hyperinsulinemia results in an increased fetal metabolic rate $(51,52)$ and increased oxygen demand with a compensatory increase in the red cell mass (53-55). The latter increases the utilization of iron and in severe cases causes depletion of tissue iron initially from liver, heart, and finally the brain as available fetal iron is shifted into the red cell mass $(6,18)$.

Within the chain of events that lead from maternal diabetes to iron deficiency, chronic fetal hypoxia co-occurs with iron deficiency, and maternal diabetes may also result in fetal ketonemia and fetal or neonatal hypoglycemia, all of which may also be harmful to the developing brain. Thus, in the human, it is difficult to disentangle the effects of brain iron deficiency from other metabolic effects of poorly controlled maternal diabetes. This study was designed to determine the contribution of iron deficiency per se to the long-term cognitive outcomes described in IDMs by Rizzo et al. and other investigators (48-50) by dividing the infants on the basis of their iron status at birth.

Of note in this study, the BIS group also had evidence of lack of adequate maternal glycemic control such that $36 \%$ of the infants were macrosomic at birth. Nevertheless, the ERPs recorded in the BIS group revealed findings that are similar to responses previously described in healthy newborn infants (14, 15) as well as normal scores on the 1-y Bayley Scales of Infant Development. This suggests that brain iron deficiency plays a significant role in the ERP differences seen between the groups in our study. The difference in iron status seen between the groups could have been due to impairment of iron transport across the placenta in the BID group as a result of longstanding vascular changes in the placenta (56) or to duration of poor maternal control. 
Ultimately, we speculate that iron deficiency represents a significant morbidity in IDMs and can result in impairments of cognitive function that is evident at birth. These impairments likely are based in brain structures that are vulnerable to iron deficiency in late gestation and are a function of maternal glycemic control. Long-term follow-up will determine whether the abnormalities seen in the BID group persist over time and are manifested in long-term impairments in recognition memory.

\section{REFERENCES}

1. Wigglesworth JM, Baum H 1988 Iron dependent enzymes in the brain. In: Youdim MBH (ed) Brain Iron: Neurochemical and Behavioural Aspects. Taylor \& Francis, New York, pp 25-66

2. Kretchmer N, Beard JL, Carlson S 1996 The role of nutrition in the development of normal cognition. Am J Clin Nutr 63:977-1001

3. Lozoff B, Jimenez E, Hagen J, Mollen E, Wolf AW 2000 Poorer behavioral and developmental outcome more than 10 years after treatment for iron deficiency in infancy. Pediatrics 105:E51

4. Chockalingam UM, Murphy E, Ophoven JC, Weisdorf SA, Georgieff MK 1987 Cord transferrin and ferritin values in newborn infants at risk for prenatal uteroplacental insufficiency and chronic hypoxia. J Pediatr 111:283-286

5. Georgieff MK, Landon MB, Mills MM, Hedlund BE, Faassen AE, Schmidt RL, Ophoven JJ, Widness JA 1990 Abnormal iron distribution in infants of diabetic mothers: spectrum and maternal antecedents. J Pediatr 117:455-461

6. Petry CD, Eaton MA, Wobken JD, Mills MM, Johnson DE, Georgieff MK 1992 Iron deficiency of liver, heart and brain in newborn infants of diabetic mothers. J Pediat 121:109-114

7. Georgieff MK, Petry CD, Wobken JD, Oyser CE 1996 Liver and brain iron deficiency in newborn infants with bilateral renal agenesis (Potter's syndrome). Pediatr Pathol Lab Med 16:509-519

8. deUngria M, Rao R, Wobken JD, Luciana M, Georgieff MK 2000 Perinatal iron deficiency decreases cytochrome c oxidase (CytOx) activity in selective regions of neonatal rat brain. Pediatr Res 48:169-176

9. Rao R, Tkac I, Townsend EL, Gruetteer R, Georgieff MK 2003 Perinatal iron deficiency alters the neurochemical profile of the developing rat hippocampus. J Nut 133:3215-3221

10. Jorgenson LA, Wobken JD, Georgieff MK 2003 Perinatal iron deficiency alters dendritic morphology and glutamate receptor expression in the developing hippocampus. Pediatr Res 53:25A

11. Seress L 2001 Morphological changes of the human hippocampal formation from midgestation to early childhood. In: Nelson CA, Luciana M (eds) Handbook of Developmental Cognitive Neuroscience. MIT Press, Cambridge, pp 45-58

12. DeCasper AJ, Fifer WP 2081980 Of human bonding: newborns prefer their mothers voices. Science 1174-1176

13. Kisilevsky BS, Hains SM, Lee K, Xie X, Huang H, Ye HH, Zhang K, Wang Z 2003 Effects of experience on fetal voice recognition. Psychol Sci 14:220-224

14. deRegnier RA, Nelson CA, Thomas K, Wewerka S, Georgieff MK 2000 Neurophysiologic evaluation of auditory recognition memory in healthy newborn infants and infants of diabetic mothers. J Pediatr 137:777-784

15. deRegnier R, Wewerka S, Georgieff MK, Mattia F, Nelson CA 2002 Influences of post-conceptional age and postnatal experience on the development of auditory recognition memory in the newborn infant. Dev Psychobiol 41:216-225

16. Nelson CA, Wewerka S, Thomas KM, Tribby-Walbridge S, deRegnier R, Georgieff MK 2000 Neurocognitive sequelae of Infants of diabetic mothers. Behav Neurosci 114:950-956

17. Nelson CA, Wewerka SS, Borscheid AJ, deRegnier RA, Georgieff MK 2003 Electrophysiologic evidence of impaired cross-modal recognition memory in 8-month-old infants of diabetic mothers. J Pediatr 142:575-582

18. Georgieff MK, Schmidt RL, Radmer WJ, Mills MM, Widness JA 1992 Tissue iron status following fetal erythropoietin stimulation. Am J Physiol 262:R405-R491

19. Saarinen UM, Siimes MA 1979 Iron absorption from breast milk, cows milk, an iron-supplemented formula: an opportunistic use of changes in total body iron determined by hemoglobin, ferritin and body weight in 132 infants. Pediatr Res 13:143-147

20. Prechtl HF 1967 Neurologic sequelae of prenatal and perinatal complications. BMJ 4:763-767

21. Nelson CA 1994 Neural correlates of recognition memory in the first postnatal year of life. In: Dawson G, Fischer K (eds) Human Behavior and the Developing Brain. Guilford Press, New York, pp 269-313

22. Novak GP, Kurtzberg D, Kreuzer JA, Vaughan HG Jr 1989 Cortical responses to speech sounds and their formants in normal infants: maturational sequence and spatiotemporal analysis. Electrencephalogr Clin Neurophysiol 73:295-305

23. Thomas DG, Crow CD 1994 Development of evoked electrical brain activity in infancy. In: Dawson G, Fischer K (eds) Human Behavior and the Developing Brain. Guilford Press, New York, pp 207-231
24. Thoman EB 1990 Sleeping and waking states in infants: a functional perspective. Neurosci Biobehav Rev 14:93-107

25. Ahonniska J, Cantell M, Tolvanen A, Lyytinen H 1993 Speech perception and brain laterality: the effect of ear advantage on auditory event-related potentials. Brain Lang $45: 127-146$

26. Jasper HH 1958 The ten-twenty electrode system of the international federation. Electroencephalogr Clin Neurophysiol 10:371-375

27. Bayley N 1993 Bayley Scales of Infant Development, 2nd Ed. The Psychological Corporation, San Antonio

28. Georgieff MK, Wewerka SW, Nelson CA, deRegnier R 2002 Iron status at 9 month of infants with low iron stores at birth. J Pediatr 141:405-409

29. Roncagliolo M, Garrido M, Walter T, Peirano P, Lozoff B 1998 Evidence of altered central nervous system development in infants with iron deficiency anemia at $6 \mathrm{mo}$ delayed maturation of auditory brain stem responses. Am J Clin Nutr 68:683-690

30. Angulo-Kinzler RM, Peirano P, Lin E, Algarin C, Garrido M, Lozoff B 2002 Twenty four hour motor activity in human infants with and without iron deficiency anemia. Early Hum Dev 70:85-101

31. Angulo-Kinzler RM, Peirano P, Lin E, Garrido M, Lozoff B 2002 Spontaneous motor activity in human infants with iron deficiency anemia. Early Hum Dev 66:67-69

32. Nelson CA 2002 Neural development and life-long plasticity. In: Lerner RM, Jacobs F, Wetlieb D (eds) Promoting Positive Child, Adolescent, and Family Development: Handbook of Program and Policy Interventions. Sage Publications, Thousand Oaks, pp 31-60

33. Felt BT, Lozoff B 1996 Brain iron and behavior of rats are not normalized by treatment of iron deficiency anemia during early development. J Nutr 126:693-701

34. Yehuda S, Youdim ME, Mostofsky DI 1986 Brain iron-deficiency causes reduced learning capacity in rats. Pharmacol Biochem Behav 25:141-144

35. Kwik-Uribe CL, Golub MS, Keen CL 2000 Chronic marginal iron intakes during early development in mice alters brain iron concentration and behavior despite postnatal iron supplementation. J Nutr 130:2040-2048

36. Eggermont JJ 1988 On the rate of maturation of sensory evoked potentials. Electroencephalogr Clin Neurophysiol 70:293-305

37. Thomas DG, Lykins MS 1995 Event-related potential measures of 24-hour retention in 5-month old infants. Dev Psychol 31:946-957

38. Dallman PR 1986 Biochemical basis for the manifestations of iron deficiency. Annu Rev Nutr 6:13-40

39. Connor JR, Menzies SL 1990 Altered cellular distribution of iron in the central nervous system of myelin deficient rats. Neuroscience 34:265-271

40. Yu GS, Steinkirchner TM, Rao GA, Larkin EC 1986 Effect of prenatal iron deficiency on myelination in rat pups. Am J Pathol 125:620-624

41. Youdim MB, Green AR, Bloomfield MR, Mitchell BD, Heal DJ, Grahame-Smith DG 1980 The effects of iron deficiency on brain biogenic monoamine biochemistry and function in rats. Neuropharmacology 19:259-267

42. Youdim MB, Yehuda S 2000 The neurochemical basis of cognitive deficits induced by brain iron deficiency: involvement of dopamine-opiate system. Cell Mol Biol 46:491-500

43. Beard JL, Chen Q, Connor J, Jones BC 1994 Altered monoamine metabolism in caudate-putamen of iron -deficient rats. Pharmacol Biochem Behav 48:621-624

44. Piqero DJ, Jones BC, Beard JL 2001 Variations in dietary iron alter behavior in developing rats. J Nutr 131:311-318

45. Tamura T, Goldenberg R, Hou J, Johnston K, Cliver SP, Ramsey SL, Nelson KG 2002 Cord serum ferritin concentrations and mental and psychomotor development of children at five years of age. J Pediatr 140:165-170

46. Algarin C, Peirano P, Garrido M, Pizarro F, Lozoff B 2003 Iron deficiency anemia in infancy: long-lasting effects on auditory and visual system functioning. Pediatr Res 53:217-223

47. Lozoff B 2000 Perinatal iron deficiency and the developing brain. Pediatr Res 48:137-139

48. Ornoy A, Wolf A, Ratzon N, Greenbaum C, Dulitzky M 1999 Neurodevelopmental outcome at early school age of children born to mothers with gestational diabetes. Arch Dis Child Fetal Neonatal Ed 81:F10-F14

49. Rizzo TA, Metzer BE, Burns WJ, Burns K 1991 Correlations between antepartum maternal metabolism and child intelligence. N Engl J Med 325:911-916

50. Rizzo TA, Ogata ES, Dooley SL 1994 Perinatal complications and cognitive development in 2 to 5 year old children of diabetic mothers. Am J Obstet Gynaecol 171:706-713

51. Milley JR, Papacostas JS, Tabata BK 1986 Effect of insulin on uptake of metabolic substrates by the sheep fetus. Am J Physiol 251:E349-E359

52. Phillips AF, Porte PJ, Stabinsky S, Rosenkrantz TS, Raye JR 1984 Effects of chronic fetal hyperglycemia upon oxygen consumption in the ovine uterus and conceptus. J Clin Invest 74:279-286

53. Stonestreet BS, Goldstein M, Oh W, Widness JA 1989 Effects of prolonged hyperinsulinemia on erythropoiesis in fetal sheep. Am J Physiol 257:R1199-R1204

54. Georgieff MK, Widness JA, Mills MM, Stonestreet BS 1989 The effect of prolonged intrauterine hyperinsulinemia on iron utilization in fetal sheep. Pediatr Res 26:467469

55. Widness JA, Susa JB, Garcia JF, Singer DB, Sehgal P, Oh W, Schwartz R, Schwartz HC 1981 Increased erythropoiesis and elevated erythropoietin in infants born to diabetic mothers and in hyperinsulinemic rhesus fetuses. J Clin Invest 67:637-642

56. Georgieff MK, Petry CD, Mills MM, McKay H, Wobken JD 1997 Increased $\mathrm{N}$-glycosylation and reduced transferrin-binding capacity of transferrin receptor isolated from placentae of diabetic women. Placenta 18:563-568 